\title{
Ion-Generating and Ion-Capturing Nanomaterials in Liquid Crystals ${ }^{\dagger}$
}

\author{
Yuriy Garbovskiy \\ UCCS BioFrontiers Center and Department of Physics, University of Colorado Colorado Springs, \\ Colorado Springs, CO 80918, USA; ygarbovs@uccs.edu or ygarbovskiy@gmail.com; Tel.: +1-719-255-3123 \\ + Presented at the 1st International Electronic Conference on Crystals, 21-31 May 2018. Available online: \\ https://sciforum.net/conference/IECC_2018.
}

Published: 21 May 2018

\begin{abstract}
The majority of tunable liquid crystal devices are driven by electric fields. The performance of such devices can be altered by the presence of small amounts of ions in liquid crystals. Therefore, the understanding of possible sources of ions in liquid crystal materials is very critical to a broad range of existing and future applications employing liquid crystals. Recently, nanomaterials in liquid crystals have emerged as a hot research topic, promising for its implementation in the design of wearable and tunable liquid crystal devices. An analysis of published results revealed that nanodopants in liquid crystals can act as either ion-capturing agents or ion-generating objects. In this presentation, a recently developed model of contaminated nanomaterials is analyzed. Nanoparticle-enabled ion capturing and ion generation regimes in liquid crystals are discussed within the framework of the proposed model. This model is in very good agreement with existing experimental results. Practical implications and future research directions are also discussed.
\end{abstract}

Keywords: liquid crystals; ions; nanomaterials; contaminated nanoparticles; ionic contamination; ion generation; ion trapping; adsorption/desorption

\section{Introduction}

A great variety of existing liquid crystal devices relies on reorientation effects when applied electric fields change the orientation of mesogenic molecules [1]. These devices include liquid crystal displays (LCD) [2], tunable optical elements such as filters [3], retarders [3], waveplates [4], and lenses [5], and optical switches [6], to name a few. The performance of the afore-mentioned devices can be altered by mobile ions, typically present in liquid crystals, through the screening effect $[2,7,8]$. In the case of liquid crystal displays, this screening effect can result in an image sticking, image flickering, reduced voltage holding ratio, and overall slow response of the display $[2,8]$. That is why it is of a paramount importance to understand possible sources of ion generation in liquid crystals [7-9].

Sources of ions in liquid crystals can be of different origins [7-10]. Ionic species can be deliberately added to liquid crystals [10-12]. Such ionic dopants (for example, tetrabutyl-ammonium tetraphenyl-boride) in liquid crystals were extensively studied back in 1970s [11,12]. Small traces of ions (metal ions and inorganic anions) in liquid crystals can originate during chemical synthesis $[13,14]$. Alignment layers and glue used to seal liquid crystal cells are also important sources of ions in liquid crystals [15-18]. External factors such as electric fields [19-22] and ionizing radiation [23,24] can enrich liquid crystals with ions. Electrochemical reactions taking place in the near-electrode areas can also generate ions in liquid crystals [25-27].

Recently, nanomaterials in liquid crystals became a hot research topic with a rapidly increasing number of publications (more details can be found in numerous review papers [28-38], and collective 
monographs [39,40]). Accumulated research data reviewed in paper [41] indicate that nanomaterials in liquid crystals can alter the behavior of ions in liquid crystals. It was reported by different research groups that carbon-based nano-objects [41-44], metal [41,45-48], dielectric [41,49-52], semiconductor $[41,53,54]$, ferroelectric [41,55-60], and other nanomaterials ([41] and references therein) can change the concentration of ions in liquid crystals. In many reported cases, nano-objects in liquid crystals can behave as ion-capturing objects thus decreasing the concentration of mobile ions in liquid crystals [41]. Interestingly, in many other cases, nanodopants in liquid crystals act as a source of ions increasing the concentration of mobile ions [41].

In an attempt to explain different, even seemingly contradictory reported results, a concept of contaminated nanomaterials was introduced [61]. In short, nanoparticles were considered contaminated with ions in liquid crystals prior to dispersing them in liquid crystals [61]. This simple approach applied to a variety of existing experimental results shows a very good agreement between the modelled and experimental data [61,62]. By dispersing contaminated nanodopants in liquid crystals, three different regimes, namely, the ion capturing regime (nanoparticles decrease the concentration of mobile ions in liquid crystals), the ion releasing or ion generation regime (nanomaterials increase the concentration of mobile ions in liquid crystals), and no change regime can be achieved [61]. The model of contaminated nanomaterials was extended to account for several types of dominant ions in liquid crystals $[63,64]$, for possible temperature-induced effects $[65,66]$, for weakly-ionized ionic species [67] and for the presence of substrates [68]. In addition, the kinetics of ion-capturing/ion releasing processes in liquid crystals doped with nanomaterials [69] and ion trapping coefficients of nanodopants [70] were also discussed.

All of these results indicate that, generally, we have to consider nanomaterials as a very important source of ions or ion traps in liquid crystals [71]. The goal of this conference paper is to summarize the most important features of the model of contaminated nanomaterials in liquid crystals [61-72] in a form of a brief tutorial accessible to a broad scientific audience.

\section{Results and Discussion}

\subsection{Model}

Consider nanoparticles in a liquid crystal host. In the most general case, these nanoparticles can be contaminated with ions prior to dispersing them in liquid crystals. To account for this ionic contamination of nanoparticles, a contamination factor $v_{N P}$ is introduced [61]. It equals a ratio of the number of surface sites of nanoparticle occupied by ionic contaminants to the total number of all surface sites of nanoparticle [61]. Typically, the number of surface sites can be characterized by their surface density $\sigma_{S}^{N P}$. Once contaminated nanoparticles are dispersed in liquid crystals, some fraction of ions can be released from their surface whereas some fraction of ions present in liquid crystals can be captured by nanoparticles. To simplify the discussion, consider the case of fully ionized ionic species characterized by their volume concentration $n=n^{+}=n^{-}$. In this case, the competition between ion-capturing and ion-releasing processes will result in the change of the concentration of mobile ions in liquid crystals doped with nanoparticles. In many practical cases, ionreleasing process can be associated with desorption of ions from nanoparticles and ion-capturing process can be described as adsorption of ions onto surface of nanoparticles. As a result, the following rate Equation (1) can be applied [69]:

$$
\frac{d n}{d t}=-k_{a}^{N P} n_{N P} A_{N P} \sigma_{S}^{N P} n\left(1-\Theta_{N P}\right)+k_{d}^{N P} n_{N P} A_{N P} \sigma_{S}^{N P} \Theta_{N P}
$$

In this equation, $n$ is the concentration of mobile ions in liquid crystals doped with nanoparticles; $t$ denotes time; $n_{N P}$ is the volume concentration of nanoparticles in liquid crystals; $\sigma_{S}^{N P}$ is the afore-mentioned surface density of all adsorption sites of a single nanoparticle; $A_{N P}$ is its surface area (for simplicity, spherical nanoparticles of a radius $R_{N P}$ are assumed); $\Theta_{N P}$ is the 
fractional surface coverage of nanoparticles; $k_{a}^{N P}$ is the adsorption rate constant; and $k_{d}^{N P}$ is the desorption rate constant. In the majority of reported experimental studies, weight concentration of nanoparticles $\omega_{N P}$ is used instead of their volume concentration $n_{N P}$. They are related as $n_{N P} \approx \omega_{N P} \frac{\rho_{L C}}{\rho_{N P}} \frac{1}{V_{N P}}$ where $\rho_{L C}\left(\rho_{N P}\right)$ is the density of liquid crystals (nanoparticles) and $V_{N P}$ is the volume of a single nanoparticle.

The first term of Equation (1) accounts for the ion-capturing process, whereas the second term originates from the ion-releasing phenomenon. This equation should be solved considering the conservation law of the total number of ions (Equation (2)):

$$
n_{0}+n_{N P} A_{N P} \sigma_{S}^{N P} v_{N P}=n+n_{N P} A_{N P} \sigma_{S}^{N P} \Theta_{N P}
$$

In Equation (2), $n_{0}$ is the initial concentration of mobile ions in liquid crystals (prior to doping them with nanomaterials); and $v_{N P}$ is the afore-mentioned contamination factor of nanoparticles. It accounts for possible contamination of nanodopants with ions [61].

It should be stressed that Equation (1) is an approximation which can be applied to liquid crystals doped with nanoparticles with certain restrictions discussed in recent papers $[64,67,72]$. In a general case, a more rigorous approach based on Boltzmann-Poisson equation should be considered [73-76].

Equations (1) and (2) can also be generalized to account for several types of dominant ions in liquid crystals $[63,64]$. In the simplest case of two dominant types of fully ionized ionic species characterized by their volume concentrations $n_{1}=n_{1}^{+}=n_{1}^{-}$and $n_{2}=n_{2}^{+}=n_{2}^{-}$, the system of Equations (3) and (4) can be used ( $j=1,2$; the meaning of physical quantities entering these equations are similar to that of Equations (1) and (2) $[61,63,64])$ :

$$
\begin{gathered}
\frac{d n_{j}}{d t}=-k_{a j}^{N P} n_{j} n_{N P} A_{N P} \sigma_{S j}^{N P}\left(1-\Theta_{N P 1}-\Theta_{N P 2}\right)+k_{d j}^{N P} n_{N P} A_{N P} \sigma_{S j}^{N P} \Theta_{N P j}^{ \pm} \\
n_{0 j}+n_{N P} \sigma_{S j}^{N P} A_{N P} v_{N P j}=n_{j}+n_{N P} \sigma_{S j}^{N P} A_{N P} \Theta_{N P j}
\end{gathered}
$$

\subsection{Kinetics of Ion-Capturing and Ion-Releasing Processes}

The kinetics of ion-capturing and ion-releasing processes in liquid crystals doped with nanoparticles was analyzed in a recent paper [69]. This analysis was based on Equations (1) and (2) and the results are shown in Figure 1 [69].

According to Figure 1a, depending on the level of ionic contamination of nanoparticles, three different regimes can be achieved: the ion releasing regime, $d n / d t>0$ (dashed-dotted-dotted, short-dashed, and short-dotted curves); ion capturing regime, $d n / d t<0$ (dotted, dashed, and dashed-dotted curves); and no change regime, $d n / d t \equiv 0$ (solid curve). The ionic contamination of nanoparticles quantified by the contamination factor $v_{N P}$ governs the switching between these regimes. The ion releasing regime is observed if $v_{N P}>v_{N P}^{C}$, the ion capturing regime holds true if $v_{N P}<v_{N P}^{C}$, and no change regime is reached if $v_{N P}=v_{N P}^{C}$, where $v_{N P}^{C}$ is the critical contamination factor of nanoparticles. It is defined as $v_{N P}^{C}=\frac{n_{0} K_{N P}}{1+n_{0} K_{N P}}$ where $K_{N P}=k_{a}^{N P} / k_{d}^{N P}$ [69]. Figure 1a also indicate that both ion capturing and ion releasing regimes depend on the concentration of nanoparticles: they are more pronounced if higher concentrations $\omega_{N P}$ are used. 

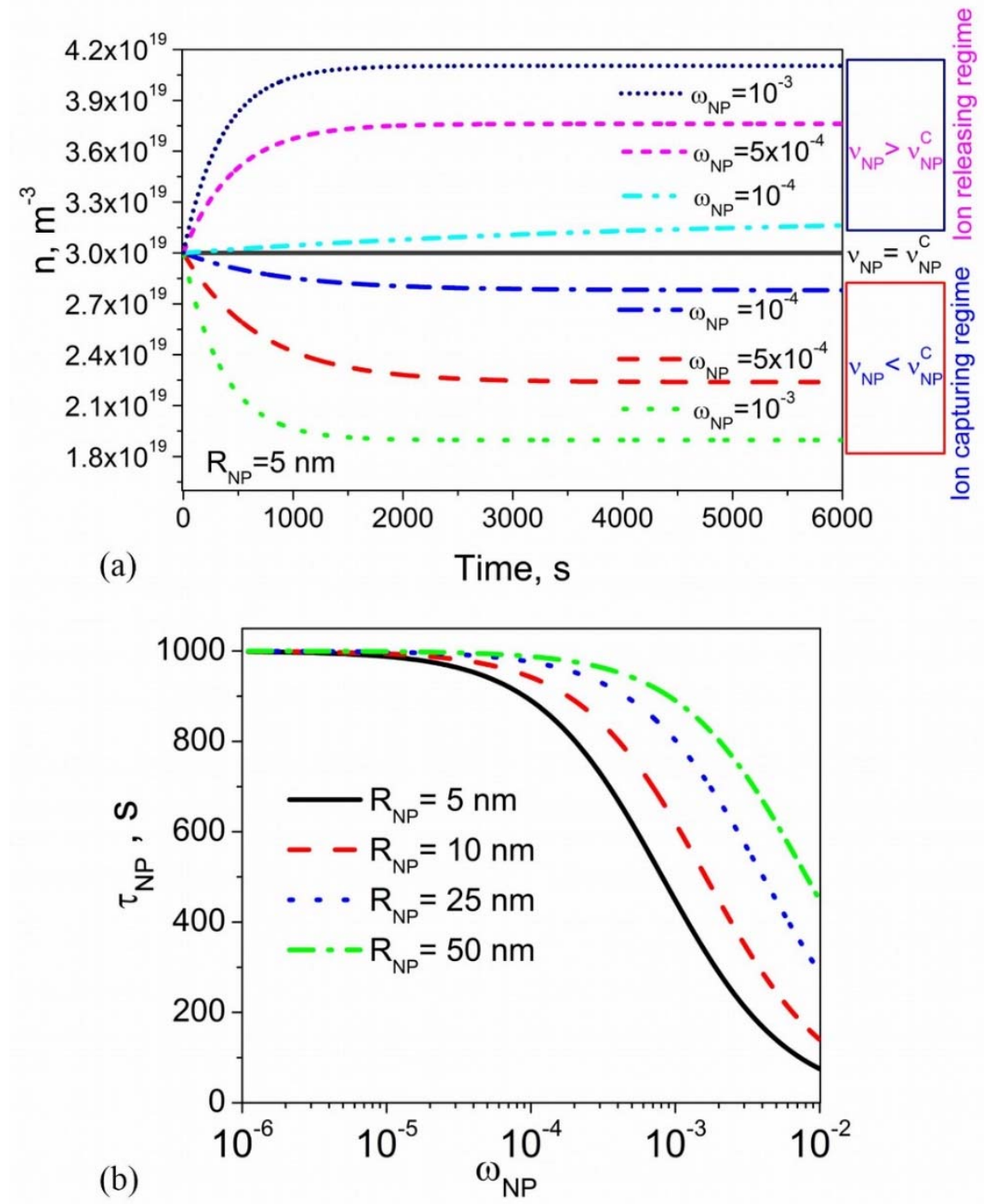

Figure 1. (a) The volume concentration of mobile ions $n$ versus time calculated using different values of the weight concentration of nanoparticles $\omega_{N P}$ and their contamination factor $v_{N P}$ ( $v_{N P}=10^{-4}$ (dotted, dashed, and dotted-dashed curves); $v_{N P}=3 \times 10^{-4}$ (solid curve); $v_{N P}=5 \times 10^{-4}$ (dashed-dotted-dotted, short-dashed, and short-dotted curves)). The radius of nanoparticles $R_{N P}$ is $5 \mathrm{~nm}$. (b) The time constant $\tau_{N P}$ as a function of the weight concentration of nanoparticles $\omega_{N P}$ calculated at different values of the nanoparticle radius $R_{N P}\left(R_{N P}=5 \mathrm{~nm}\right.$ (dashed-dotted curve); $R_{N P}=10 \mathrm{~nm}$ (dashed curve); $R_{N P}=25 \mathrm{~nm} \quad$ (dotted curve); $R_{N P}=50 \mathrm{~nm}$ (solid curve)). Other parameters used in simulations: $K_{N P}=10^{-23} \mathrm{~m}^{3}$, $k_{d}^{N P}=10^{-3} \mathrm{~s}^{-1}, \sigma_{S}^{N P}=0.8 \times 10^{18} \mathrm{~m}^{-2}, n_{0}=3 \times 10^{19} \mathrm{~m}^{-3}, \rho_{N P} / \rho_{L C}=3.9$. Reproduced from Nanomaterials 2018, 8(2), 59; https://doi.org/10.3390/nano8020059 [69], under the Creative Commons Attribution License.

The time constant $\tau_{N P}$ characterizing the kinetics of ion-capturing/ion-releasing process shown in Figure 1a can be defined through Equation (5):

$$
n\left(\tau_{N P}\right)-n_{0}=(1-1 / e)\left(n_{\infty}-n_{0}\right)
$$

where $n_{0}=n(t=0)$ and $n_{\infty}=n(t \rightarrow \infty)$. In the regime of low surface coverage $\left(\Theta_{N P}<<1\right)$ this time constant is given by Equation (6): 


$$
\tau_{N P}=1 / k_{d}^{N P}\left(K_{N P} n_{N P} A_{N P} \sigma_{S}^{N P}+1\right)
$$

In the case of spherical nanoparticles of radius $R_{N P}$, the dependence of the time constant on the weight concentration of nanodopants is shown in Figure $1 \mathrm{~b}$. As can be seen, by using smaller nanoparticles and their higher concentrations one can decrease time needed to achieve the steadystate. However, it should be noted that this decrease is diffusion-limited. In other words, Equation (6) is correct as long as $\tau_{N P} \gg \tau_{D}$. The characteristic time $\tau_{D}$ can be estimated by means of Equation (7):

$$
\tau_{D}=\frac{l_{D}^{2}}{6 D} \approx \frac{1}{6 D \sqrt[3]{n^{2}}}
$$

where $l_{D}$ is the average distance between mobile ions in liquid crystals, and $D$ is the diffusion coefficient of ions. By using typical values $\left(n \approx 10^{20} \mathrm{~m}^{-3}\right.$ and $D=10^{-12} \mathrm{~m}^{2} / \mathrm{s}$ [13] ) this time can be estimated as $\tau_{D} \approx 8 \times 10^{-3}$ s. By comparing it to data shown in Figure $1 \mathrm{~b}$ it can be seen that, indeed, $\tau_{N P}>\tau_{D}$.

\subsection{Steady-State Regime}

In the majority of the reported experimental studies, steady-state measurements are performed $\left(\frac{d n}{d t}=0\right)$. In regard to the concentration of mobile ions in liquid crystals doped with nanomaterials, an analysis of possible regimes achieved in such systems was done in paper [61]. Three regimes, namely, the ion capturing regime (solid curve), ion releasing regime (dashed curve), and no change regime (dotted curve) are shown in Figure 2 where the concentration of mobile ions in liquid crystals is plotted as a function of the weight concentration of nanoparticles.

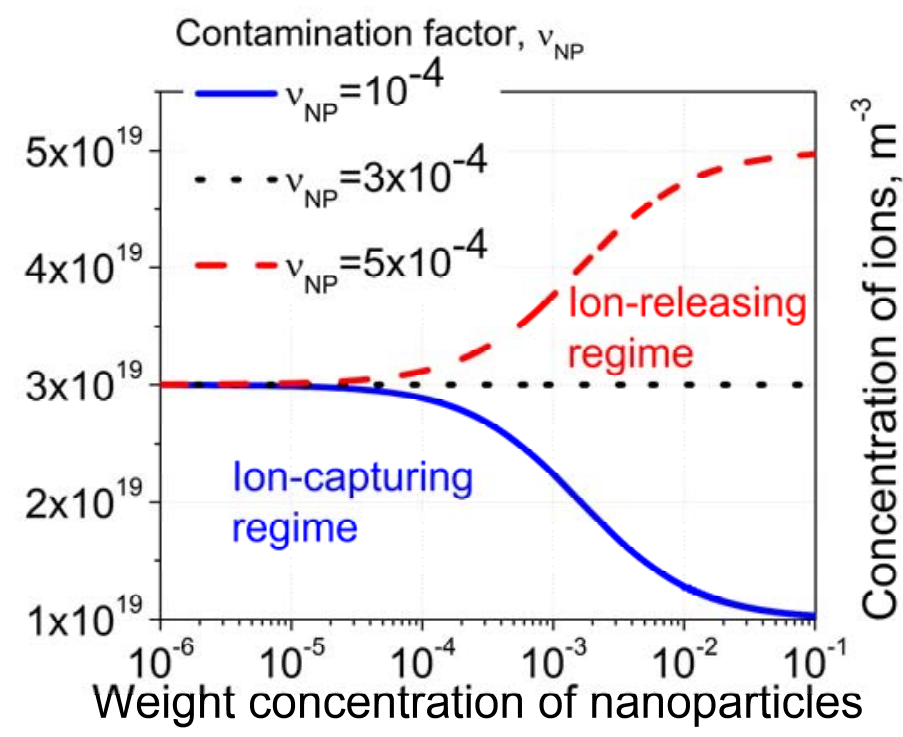

Figure 2. The volume concentration of mobile ions $n$ in liquid crystals versus the weight concentration of nanoparticles $\omega_{N P}$ calculated at different values of their contamination factor $v_{N P}\left(v_{N P}=10^{-4}\right.$ (solid curve); $v_{N P}=3 \times 10^{-4}$ (dotted curve); and $v_{N P}=5 \times 10^{-4}$ (dashed curve)). The radius of nanoparticles $R_{N P}$ is $10 \mathrm{~nm}$. Other parameters used in simulations: $K_{N P}=10^{-23} \mathrm{~m}^{3}, \sigma_{S}^{N P}=0.8 \times 10^{18} \mathrm{~m}^{-2}, n_{0}=3 \times 10^{19} \mathrm{~m}^{-3}, \rho_{N P} / \rho_{L C}=3.9$. This image is also posted on Nanowerk Spotlight [77]. 
In the case of ion capturing regime, the concentration of mobile ions in liquid crystals decreases as the weight concentration of nanodopants goes up $\left(d n / d \omega_{N P}<0\right)$. This regime is achieved if $v_{N P}<v_{N P}^{C}$. The ion releasing regime is characterized by the increase in the concentration of mobile ions with an increase in the weight concentration of nanoparticles $\left(d n / d \omega_{N P}>0\right)$. It is observed if $v_{N P}>v_{N P}^{C}$. The concentration of mobile ions in liquid crystals doped with nanoparticles does not change if $v_{N P}=v_{N P}^{C}$. Switching between these three different regimes can be achieved by changing the level of ionic contamination of nanomaterials $v_{N P}$, the ionic purity of liquid crystals (an initial concentration of mobile ions $n_{0}$ ), and by varying materials used in experiments (constant $K_{N P}=k_{a}^{N P} / k_{d}^{N P}$ ) as shown in Table 1 (this table is created using similar table published in paper [61]).

Table 1. Ion-capturing, ion-releasing, and no change regimes in liquid crystals doped with contaminated nanoparticles [61].

\begin{tabular}{ccccc}
\hline & $\begin{array}{c}\text { Ion-Capturing } \\
\text { Regime }\end{array}$ & No Change Regime & $\begin{array}{c}\text { Ion-Releasing } \\
\text { Regime }\end{array}$ \\
\hline $\begin{array}{c}\text { Contamination level of } \\
\text { nanomaterials, } v_{N P}\end{array}$ & $v_{N P}<\frac{K_{N P} n_{0}}{1+K_{N P} n_{0}}$ & $v_{N P}=\frac{K_{N P} n_{0}}{1+K_{N P} n_{0}}$ & $v_{N P}>\frac{K_{N P} n_{0}}{1+K_{N P} n_{0}}$ \\
\hline $\begin{array}{c}\text { Initial concentration of } \\
\text { ions in liquid crystals, } \\
n_{0}\end{array}$ & $n_{0}>\frac{1}{K_{N P}\left(\frac{1}{v_{N P}}-1\right)}$ & $n_{0}=\frac{1}{K_{N P}\left(\frac{1}{v_{N P}}-1\right)}$ & $n_{0}<\frac{1}{K_{N P}\left(\frac{1}{v_{N P}}-1\right)}$ \\
\hline Constant, $K_{N P}$ & $K_{N P}>\frac{1}{n_{0}\left(\frac{1}{v_{N P}}-1\right)}$ & $K_{N P}=\frac{1}{n_{0}\left(\frac{1}{v_{N P}}-1\right)}$ & $K_{N P}<\frac{1}{n_{0}\left(\frac{1}{v_{N P}}-1\right)}$ \\
\hline
\end{tabular}

\subsection{Temperature-Induced Effects}

Constants describing ion-capturing $\left(k_{a}^{N P}\right)$ and ion-releasing $\left(k_{d}^{N P}\right)$ processes in liquid crystals doped with nanomaterials are temperature-dependent $[65,66]$. By approximating this temperature dependence through Equations (8) and (9), temperature-induced ionic effects in liquid crystals doped with nanoparticles can be analyzed $[65,66]$ :

$$
\begin{aligned}
& k_{a}^{N P}=k_{a}^{0} e^{-E_{a} / k T} \\
& k_{d}^{N P}=k_{d}^{0} e^{-E_{d} / k T}
\end{aligned}
$$

where $E_{a}$ is the adsorption activation energy; $E_{d}$ is the desorption activation energy; $k_{a}^{0}$ and $k_{d}^{0}$ are pre-exponential factors; $k=1.38 \times 10^{-23} \mathrm{~J} / \mathrm{K}$, and $T$ is temperature $[65,66]$.

By applying Equations (8) and (9), constant $K_{N P}$ can be written as expression (10):

$$
K_{N P}=\frac{k_{a}^{N P}}{k_{d}^{N P}}=K_{0}^{N P} e^{\Delta E / k T}
$$

In this equation, $K_{0}^{N P}=k_{a}^{0} / k_{d}^{0}$ is the pre-exponential factor, and $\Delta E=E_{d}-E_{a}[65,66]$. 
Temperature dependence $K_{N P}(T)$ (Equation (10)) can result in temperature-induced release of ions experimentally observed in liquid crystals doped with nanoparticles [65]. Typical dependence calculated using Equations (1), (2) and (10) is shown in Figure 3.
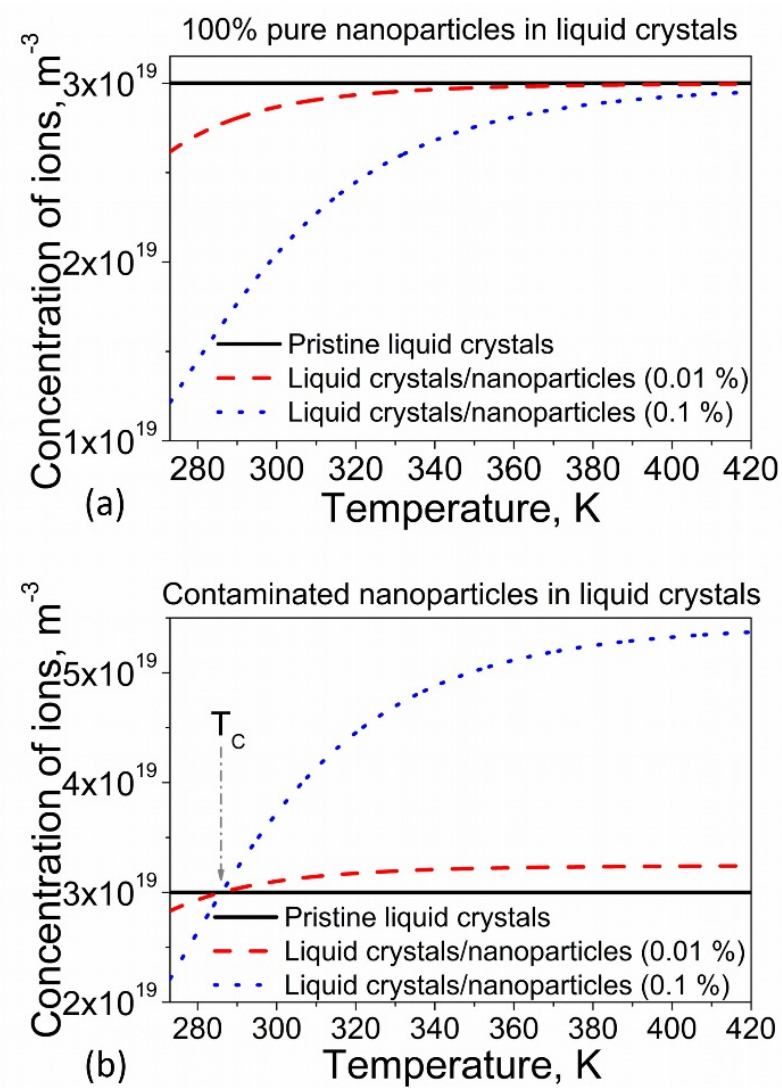

Figure 3. The volume concentration of mobile ions $n$ in liquid crystals doped with nanoparticles plotted as a function of temperature for two cases: (a) 100\% pure nanoparticles in liquid crystals; and (b) contaminated nanoparticles in liquid crystals. Physical parameters used in simulations: $v_{N P}=0$

(a) and $\quad v_{N P}=4 \times 10^{-4} \quad(\mathbf{b}) ; \quad K_{N P}(T=293 K)=10^{-23} \mathrm{~m}^{3} \quad ; \quad \Delta E=+0.3 \quad \mathrm{eV}$; $\sigma_{S}^{N P}=0.8 \times 10^{18} \mathrm{~m}^{-2} ; n_{0}=3 \times 10^{19} \mathrm{~m}^{-3} ; \rho_{N P} / \rho_{L C}=3.9$. The radius of nanoparticles $R_{N P}$ is $10 \mathrm{~nm}$. The weight concentration of nanoparticles is $0.01 \%$ (dashed curve) and $0.1 \%$ (dotted curve). This image is also posted on Nanowerk Spotlight [78].

Figure 3a illustrates the so-called temperature-induced release of ions in liquid crystals doped with nanoparticles. The concentration of mobile ions in liquid crystals doped with nanomaterials increases as its temperature goes up. In the case of $100 \%$ pure nanodopants, this increase saturates at higher temperatures approaching an initial concentration of ions in liquid crystals (it means at high enough temperature nanoparticles lose their ion-capturing properties, see Figure 3a). It should be stressed that if $100 \%$ pure nanoparticles are mixed with liquid crystals, the concentration of mobile ions in such systems is always less or equal the initial concentration: $n(T) \leq n_{0}$. In other words, the ion-capturing regimes is observed (and it approaches the "no change" regime $\left(n(T) \rightarrow n_{0}\right)$ at elevated temperatures, Figure 3a). On a contrary, the $n(T)$ dependence of liquid crystals doped with contaminated nanomaterials, exhibits some interesting features (Figure 3b). There are two distinct regions (Figure $3 b$ ). At temperatures $T<T_{C}$ the concentration of mobile ions in liquid crystals doped with nanomaterials is less than the concentration of ions in pristine (without nanodopants) liquid crystals $\left(n(T)<n_{0}\right)$ which corresponds to the ion-capturing regime. Above this 
temperature ( $T>T_{C}$ ), an opposite inequality holds true $n(T)>n_{0}$ which corresponds to the ionreleasing regime (Figure $3 b$ ). No change regime corresponds to temperature $T_{C}$. Temperature $T_{C}$ can be found using Equation (11) [65]:

$$
n_{0}=\frac{v_{N P}}{K_{N P}\left(T_{C}\right)\left(1-v_{N P}\right)}
$$

Thus, a temperature-induced switching between ion-capturing and ion-releasing regimes can be achieved in liquid crystals doped with contaminated nanomaterials [65].

Temperature-induced release of ions is observed in systems characterized by positive values of their parameter $\Delta E>0$. Interestingly, liquid crystals doped with nanoparticles and characterized by negative values of this parameter $(\Delta E<0)$ should exhibit an opposite effect, namely, temperature-induced capturing of ions [66]. This unusual effect was analyzed in paper [66].

\section{Case Studies: A Brief Survey}

The proposed model of contaminated nanoparticles in liquid crystals [61] was successfully applied to existing experimental data [62,71]. Table 2 provides a summary of the observed experimental effects and physical parameters used in calculations to achieve a very good agreement between the model and experiments.

Table 2. Case studies: reported experimental data and physical parameters of the model.

\begin{tabular}{|c|c|c|}
\hline Materials & Reported Effects & Physical Parameters \\
\hline $\begin{array}{c}\text { Anatase }\left(\mathrm{TiO}_{2}\right) \\
\text { nanoparticles in nematic } \\
\text { liquid crystals (E44) }\end{array}$ & $\begin{array}{c}\text { Ion capturing effect } \\
{[49]}\end{array}$ & $\begin{array}{c}K_{N P}=10^{-23} \mathrm{~m}^{3} ; v_{N P}=1.5 \times 10^{-4} ; \\
\sigma_{S}^{N P}=0.8 \times 10^{18} \mathrm{~m}^{-2} ; R_{N P}=5 \mathrm{~nm} ; \\
\rho_{N P} / \rho_{L C}=3.9[62]\end{array}$ \\
\hline $\begin{array}{c}\text { Carbon nanotubes (CNT) } \\
\text { in nematic liquid crystals } \\
\text { (E7) }\end{array}$ & $\begin{array}{c}\text { Ion capturing effect } \\
{[42]}\end{array}$ & $\begin{array}{c}K_{N P}=0.7 \times 10^{-23} \mathrm{~m}^{3} ; v_{N P}=9.5 \times 10^{-6} ; \\
\sigma_{S}^{N P}=10^{18} \mathrm{~m}^{-2} ; R_{C N T}=2.5 \mathrm{~nm} ; \\
L_{C N T}=500 \mathrm{~nm} \rho_{N P} / \rho_{L C}=1.6\end{array}$ \\
\hline $\begin{array}{l}\text { Diamond nanoparticles in } \\
\text { nematic liquid crystals (E7) }\end{array}$ & $\begin{array}{c}\text { Ion capturing effect } \\
\text { [43] }\end{array}$ & $\begin{array}{c}K_{N P}=10^{-22} \mathrm{~m}^{3} ; v_{N P}=10^{-2} \\
\sigma_{S}^{N P}=1.25 \times 10^{17} \mathrm{~m}^{-2} ; R_{N P}=5 \mathrm{~nm} ; \\
\rho_{N P} / \rho_{L C}=3.3[62]\end{array}$ \\
\hline $\begin{array}{l}\text { Diamond nanoparticles in } \\
\text { nematic liquid crystals (E7) }\end{array}$ & $\begin{array}{c}\text { Ion releasing effect } \\
{[43]}\end{array}$ & $\begin{array}{c}K_{N P}=0.8 \times 10^{-25} \mathrm{~m}^{3} ; v_{N P}=0.25 \\
\sigma_{S}^{N P}=1.25 \times 10^{17} \mathrm{~m}^{-2} ; R_{N P}=5 \mathrm{~nm} ; \\
\rho_{N P} / \rho_{L C}=3.3[62]\end{array}$ \\
\hline $\begin{array}{l}\text { Graphene nano-flakes } \\
\text { (GNF) in nematic liquid } \\
\text { crystals (8OCB) }\end{array}$ & $\begin{array}{c}\text { Ion capturing effect } \\
{[79]}\end{array}$ & $\begin{array}{c}K_{N P}=0.8 \times 10^{-23} \mathrm{~m}^{3} ; v_{N P}=8.5 \times 10^{-6} ; \\
\sigma_{S}^{N P}=0.33 \times 10^{18} \mathrm{~m}^{-2} ; R_{G N F}=5 \mathrm{~nm} ; \\
L_{G N F}=10 \mathrm{~nm} ; \rho_{N P} / \rho_{L C}=1.8\end{array}$ \\
\hline
\end{tabular}




\begin{tabular}{|c|c|c|}
\hline $\begin{array}{c}\text { Ferroelectric nanoparticles } \\
\qquad\left(\mathrm{LiNbO}_{3}\right) \text { in liquid } \\
\text { crystals }\end{array}$ & $\begin{array}{c}\text { Ion capturing effect } \\
{[55]}\end{array}$ & $\begin{array}{c}K_{N P}=7 \times 10^{-23} \mathrm{~m}^{3} ; v_{N P}=0.1075 ; \\
\sigma_{S}^{N P}=5 \times 10^{18} \mathrm{~m}^{-2} ; R_{N P}=12.5 \mathrm{~nm} ; \\
\rho_{N P} / \rho_{L C}=4.65[62]\end{array}$ \\
\hline $\begin{array}{c}\text { Ferroelectric nanoparticles } \\
\left(\mathrm{BaTiO}_{3}\right) \text { in nematic } \\
\text { liquid crystals }\end{array}$ & $\begin{array}{c}\text { Ion capturing effect } \\
{[57]}\end{array}$ & $\begin{array}{c}K_{N P}=4 \times 10^{-20} \mathrm{~m}^{3} ; v_{N P}=0.3 \\
\sigma_{S}^{N P}=10^{19} \mathrm{~m}^{-2} ; R_{N P}=1000 \mathrm{~nm} ; \\
\rho_{N P} / \rho_{L C}=6.02\end{array}$ \\
\hline $\begin{array}{l}\text { Ferroelectric nanoparticles } \\
\left.\qquad \mathrm{BaTiO}_{3}\right) \text { in nematic } \\
\text { liquid crystals (E44) }\end{array}$ & $\begin{array}{l}\text { Temperature- } \\
\text { induced release of } \\
\text { ions [58] }\end{array}$ & $\begin{array}{c}v_{N P}=0 ; K_{0}^{N P}=1.93 \times 10^{-30} \mathrm{~m}^{3} ; \\
\Delta E=0.4 \mathrm{eV} ; \sigma_{S}^{N P}=5 \times 10^{18} \mathrm{~m}^{-2} \\
R_{N P}=20 \mathrm{~nm} ; \rho_{N P} / \rho_{L C}=6.02[65]\end{array}$ \\
\hline $\begin{array}{c}\mathrm{TiO}_{2} \text { nanoparticles in } \\
\text { nematic liquid crystals } \\
\text { (ZhK1282) }\end{array}$ & $\begin{array}{c}\text { Ion releasing effect } \\
{[51]}\end{array}$ & $\begin{array}{c}v_{N P}=4.35 \times 10^{-4} ; K_{N P}=1.6 \times 10^{-23} \mathrm{~m}^{3} ; \\
\sigma_{S}^{N P}=0.8 \times 10^{18} \mathrm{~m}^{-2} ; R_{N P}=25 \mathrm{~nm} ; \\
\rho_{N P} / \rho_{L C}=3.9\end{array}$ \\
\hline $\begin{array}{c}\mathrm{TiO}_{2} \text { nanoparticles in } \\
\text { nematic liquid crystals } \\
\text { (ZhK1282) }\end{array}$ & $\begin{array}{c}\text { Ion capturing effect } \\
{[51]}\end{array}$ & $\begin{array}{c}v_{N P}=0 ; K_{N P}=3.65 \times 10^{-24} \mathrm{~m}^{3} ; \\
\sigma_{S}^{N P}=2 \times 10^{18} \mathrm{~m}^{-2} ; R_{N P}=25 \mathrm{~nm} ; \\
\rho_{N P} / \rho_{L C}=3.9\end{array}$ \\
\hline $\begin{array}{c}\text { CdSe/ZnS core/shell } \\
\text { nanoparticles in nematic } \\
\text { liquid crystals (ZhK1289) }\end{array}$ & $\begin{array}{c}\text { Ion releasing effect } \\
{[53]}\end{array}$ & $\begin{array}{c}v_{N P}=3.379 \times 10^{-3} ; K_{N P}=10^{-26} \mathrm{~m}^{3} ; \\
\sigma_{S}^{N P}=10^{18} \mathrm{~m}^{-2} ; R_{N P}=3 \mathrm{~nm} ; \\
\rho_{N P} / \rho_{L C}=5.091\end{array}$ \\
\hline $\begin{array}{c}\mathrm{Cu}_{7} P S_{6} \text { nanoparticles in } \\
\text { nematic liquid crystals } \\
(6 \mathrm{CB})\end{array}$ & $\begin{array}{c}\text { Ion releasing effect } \\
{[52]}\end{array}$ & $\begin{array}{c}v_{N P}=0.3075 ; K_{N P}=10^{-23} \mathrm{~m}^{3} ; \\
\sigma_{S}^{N P}=7 \times 10^{18} \mathrm{~m}^{-2} ; R_{N P}=58.5 \mathrm{~nm} ; \\
\rho_{N P} / \rho_{L C}=4.907[71]\end{array}$ \\
\hline
\end{tabular}

\section{Conclusions}

Existing experimental results (Table 2) unambiguously show that nanomaterials in liquid crystals can affect the concentration of ions in different ways. The dispersion of nanomaterials in liquid crystals can result in the ion capturing effect, ion releasing effect, or the combination of them. Therefore, nanomaterials in liquid crystals should be considered as new sources of ions or as ion trapping objects. The model of contaminated nanomaterials in liquid crystals reviewed in this conference paper can predict both ion capturing and ion releasing (or ion generation) regimes (Figures 1-3). Moreover, it also predicts a new effect, namely temperature-induced ion capturing effect [66]. This model is in very good agreement with reported experimental data (Table 2).

So far, the origin of ionic contamination of nanomaterials is poorly understood. In many practical cases, this contamination can originate from particular chemical procedures utilized during chemical synthesis of nano-objects. Ionic contaminants can also originate from the contact of nanomaterials with environment and due to external factors such as ionizing radiation, high electric fields, excessive heating and chemical degradation. The aforementioned possible causes of ionic contamination of nanomaterials are caused by external factors and, therefore, are extrinsic in nature. This type of ionic 
contamination is typically characterized by relatively low values of the contamination factor. It can be reduced or even eliminated by improving physical/chemical procedures used to produce, storage, and handle nanomaterials. There is also an intrinsic source of ionic contamination of nanoparticles. For example, self-dissociating nanomaterials can generate ions because of their chemical/physical composition. In this case, the contamination factor of nanoparticles is relatively high and cannot be reduced by improving the purification procedure. Interestingly, both types of ionic contamination (intrinsic and extrinsic) can be successfully analyzed by the model reviewed in this paper. Further studies are needed to understand mechanisms of ionic contamination of nanomaterials and their impact on the properties of liquid crystals.

Author Contributions: Y.G. conceived the idea of the paper, performed all simulations, and wrote the paper.

Acknowledgments: The author would like to acknowledge the support provided by the UCCS BioFrontiers Center at the University of Colorado.

Conflicts of Interest: The author declares no conflict of interest.

\section{Abbreviations}

The following abbreviations are used in this manuscript:

MDPI Multidisciplinary Digital Publishing Institute

DOAJ Directory of open access journals

LCD liquid crystal display

\section{References}

1. Yang, D.-K.; Wu, S.-T. Liquid Crystal Devices; John Wiley \& Sons: Hoboken, NJ, USA, 2006; pp. 1-378.

2. Chigrinov, V.G. Liquid Crystal Devices: Physics and Applications; Artech House: Boston, MA, USA, 1999; pp. $1-360$.

3. Abdulhalim, I. Non-display bio-optic applications of liquid crystals. Liq. Cryst. Today 2011, 20, 44-60.

4. De Sio, L.; Roberts, D.E.; Liao, Z.; Hwang, J.; Tabiryan, N.; Steeves, D.M.; Kimball, B.R. Beam shaping diffractive wave plates. Appl. Opt. 2018, 57, A118-A121.

5. Lin, Y.-H.; Wang, Y.-J.; Reshetnyak, V. Liquid crystal lenses with tunable focal length. Liq. Cryst. Rev. 2017, 5, 111-143.

6. Geis, M.W.; Bos, P.J.; Liberman, V.; Rothschild, M. Broadband optical switch based on liquid crystal dynamic scattering. Opt. Express 2016, 24, 13812-13823.

7. Naemura, S. Electrical properties of liquid crystal materials for display applications. Mater. Res. Soc. Symp. Proc. 1999, 559, 263-274.

8. Neyts, K.; Beunis, F. Ion transport in liquid crystals. In Handbook of Liquid Crystals: Physical Properties and Phase Behavior of Liquid Crystals; Wiley-VCH: Weinheim, Germany, 2014; Volume 2, Chapter 11, pp. 357382.

9. Korniychuk, P.P.; Gabovich, A.M.; Singer, K.; Voitenko, A.I.; Reznikov, Y.A. Transient and steady electric currents through a liquid crystal cell. Liq. Cryst. 2010, 37, 1171-1181.

10. Blinov, L.M. Structure and Properties of Liquid Crystals; Springer: New York, NY, USA, 2010.

11. Chang, R.; Richardson, J.M. The anisotropic electrical conductivity of MBBA containing tetrabutylammonium tetraphenyl-boride. Mol. Cryst. Liq. Cryst. 1973, 28, 189-200.

12. Barnik, M.I.; Blinov, L.M.; Grebenkin, M.F.; Pikin, S.A.; Chigrinov, V.G. Electrohydrodynamic instability in nematic liquid crystals. Sov. Phys. JETP 1976, 42, 550-553.

13. Naemura, S.; Sawada, A. Ionic conduction in nematic and smectic liquid crystals. Mol. Cryst. Liq. Cryst. 2003, 400, 79-96.

14. Hung, H.Y.; Lu, C.W.; Lee, C.Y.; Hsu, C.S.; Hsieh, Y.Z. Analysis of metal ion impurities in liquid crystals using high resolution inductively coupled plasma mass spectrometry. Anal. Methods 2012, 4, 3631-3637.

15. Murakami, S.; Naito, H. Electrode and interface polarizations in nematic liquid crystal cells. Jpn. J. Appl. Phys. 1997, 36, 2222-2225.

16. Mizusaki, M.; Enomoto, S.; Hara, Y. Generation mechanism of residual direct current voltage for liquid crystal cells with polymer layers produced from monomers. Liq. Cryst. 2017, 44, 609-617. 
17. Kravchuk, R.; Koval'chuk, O.; Yaroshchuk, O. Filling initiated processes in liquid crystal cell. Mol. Cryst. Liq. Cryst. 2002, 384, 111-119.

18. Garbovskiy, Y. Time dependent electrical properties of liquid crystal cells: Unravelling the origin of ion generation. Liq. Cryst. 2018, 45, 1540-1548, doi:10.1080/02678292.2018.1455228.

19. Chieu, T.C.; Yang, K.H. Transport properties of ions in ferroelectric liquid crystal cells. Jpn. J. Appl. Phys. 1989, 28, 2240-2246.

20. Murakami, S.; Naito, H. Charge injection and generation in nematic liquid crystal cells. Jpn. J. Appl. Phys. 1997, 36, 773-776.

21. Naemura, S.; Sawada, A. Ion Generation in Liquid Crystals under Electric Field. Mol. Cryst. Liq. Cryst. 2000, 346, 155-168.

22. De Vleeschouwer, H.; Verschueren, A.; Bougrioua, F.; Van Asselt, R.; Alexander, E.; Vermael, S.; Neyts, K.; Pauwels, H. Long-term ion transport in nematic liquid crystal displays. Jpn. J. Appl. Phys. 2001, 40, 32723276.

23. Kovalchuk, A.V.; Lavrentovich, O.D.; Linev, V.A. Electrical conductivity of $\gamma$-irradiated cholesteric liquid crystals. Sov. Tech. Phys. Lett. 1988, 14, 381-382.

24. Naito, H.; Yoshida, K.; Okuda, M.; Sugimura, A. Transient Current Study of Ultraviolet-Light-Soaked States in n-Pentyl-p-n-Cyanobiphenyl. Jpn. J. Appl. Phys. 1994, 33, 5890-5891.

25. Barret, S.; Gaspard, F.; Herino, R.; Mondon, F. Dynamic scattering in nematic liquid crystals under dc conditions. I. Basic electrochemical analysis. J. Appl. Phys. 1976, 47, 2375-2377.

26. Barret, S.; Gaspard, F.; Herino, R.; Mondon, F. Dynamic scattering in nematic liquid crystals under dc conditions. II. Monitoring of electrode processes and lifetime investigation. J. Appl. Phys. 1976, 47, 23782381.

27. Lim, H.S.; Margerum, J.D.; Graube, A. Electrochemical properties of dopants and the DC dynamic scattering of a nematic liquid crystal. J. Electrochem. Soc. Solid State Sci. Technol. 1977, 124, 1389-1394.

28. Rahman, M.; Lee, W. Scientific duo of carbon nanotubes and nematic liquid crystals. J. Phys. D Appl. Phys. 2009, 42, 063001.

29. Garbovskiy, Y.; Glushchenko, A. Liquid crystalline colloids of nanoparticles: Preparation, properties, and applications. Solid State Phys. 2011, 62, 1-74.

30. Garbovskiy, Y.; Zribi, O.; Glushchenko, A. Emerging Applications of Ferroelectric Nanoparticles in Materials Technologies, Biology and Medicine. In Advances in Ferroelectrics; Peláiz-Barranco, A., Ed.; InTech: Rijeka, Croatia, 2012; ISBN 978-953-51-0885-6, doi:10.5772/52516.

31. Mirzaei, J.; Reznikov, M.; Hegmann, T. Quantum dots as liquid crystal dopants. J. Mater. Chem. 2012, 22, 22350-22365.

32. Stamatoiu, O.; Mirzaei, J.; Feng, X.; Hegmann, T. Nanoparticles in liquid crystals and liquid crystalline nanoparticles. Top. Curr. Chem. 2012, 318, 331-394.

33. Blanc, C.; Coursault, D.; Lacaze, E. Ordering nano- and microparticles assemblies with liquid crystals. Liq. Cryst. Rev. 2013, 1, 83-109.

34. Kumar, S. Discotic liquid crystal-nanoparticle hybrid systems NPG Asia Mater. 2014, 6, e82.

35. Urbanski, M. On the impact of nanoparticle doping on the electro-optic response of nematic hosts. Liq. Cryst. Today 2015, 24, 102-115.

36. Klimusheva, G.; Mirnaya, T.; Garbovskiy, Y. Versatile Nonlinear-Optical Materials Based on Mesomorphic Metal Alkanoates: Design, Properties, and Applications. Liq. Cryst. Rev. 2015, 3, $28-57$.

37. Yadav, S.P.; Singh, S. Carbon nanotube dispersion in nematic liquid crystals: An overview. Prog. Mater. Sci. 2016, 80, 38-76.

38. Mertelj, A.; Lisjak, D. Ferromagnetic nematic liquid crystals. Liq. Cryst. Rev. 2017, 5, 1-33.

39. Li, Q. (Ed.) Nanoscience with Liquid Crystals; Springer: Cham, Switzerland, 2014; p. 420.

40. Lagerwall, J.P.F.; Scalia, G. Liquid Crystals with Nano and Microparticles; Series in Soft Condensed Matter; World Scientific Publishing Co: Singapore, 2016; Volume 7, pp. 461-920.

41. Garbovskiy, Y.; Glushchenko, I. Nano-Objects and Ions in Liquid Crystals: Ion Trapping Effect and Related Phenomena. Crystals 2015, 5, 501-533.

42. Jian, B.R.; Tang, C.Y.; Lee, W. Temperature-dependent electrical properties of dilute suspensions of carbon nanotubes in nematic liquid crystals. Carbon 2011, 49, 910-914.

43. Tomylko, S.; Yaroshchuk, O.; Kovalchuk, O.; Maschke, U.; Yamaguchi, R. Dielectric properties of nematic liquid crystal modified with diamond nanoparticles. Ukr. J. Phys. 2012, 57, 239-243. 
44. Wu, P.C.; Lisetski, L.N.; Lee, W. Suppressed ionic effect and low-frequency texture transitions in a cholesteric liquid crystal doped with graphene nanoplatelets. Opt. Express 2015, 23, 11195-11120, doi:10.1364/OE.23.011195.

45. Shukla, R.K.; Feng, X.; Umadevi, S.; Hegmann, T.; Haase, W. Influence of different amount of functionalized bulky gold nanorods dopant on the electrooptical, dielectric and optical properties of the FLC host. Chem. Phys. Lett. 2014, 599, 80-85.

46. Podgornov, F.V.; Wipf, R.; Stühn, B.; Ryzhkova, A.V.; Haase, W. Low-frequency relaxation modes in ferroelectric liquid crystal/gold nanoparticle dispersion: Impact of nanoparticle shape. Liq. Cryst. 2016, 43, 1536-1547.

47. Urbanski, M.; Lagerwall, J.P.F. Why organically functionalized nanoparticles increase the electrical conductivity of nematic liquid crystal dispersions. J. Mater. Chem. C 2017, 5, 8802-8809.

48. Podgornov, F.V.; Gavrilyak, M.; Karaawi, A.; Boronin, V.; Haase, W. Mechanism of electrooptic switching time enhancement in ferroelectric liquid crystal/gold nanoparticles dispersion. Liq. Cryst. 2018, 45, 15941602, doi:10.1080/02678292.2018.1458256.

49. Tang, C.Y.; Huang, S.M.; Lee, W. Electrical properties of nematic liquid crystals doped with anatase $\mathrm{TiO}_{2}$ nanoparticles. J. Phys. D Appl. Phys. 2011, 44, 355102.

50. Chandran, A.; Prakash, J.; Gangwar, J.; Joshi, T.; Kumar Srivastava, A.; Haranath, D.; Biradar Ashok, M. Low-voltage electro-optical memory device based on $\mathrm{NiO}$ nanorods dispersed in a ferroelectric liquid crystal. RSC Adv. 2016, 6, 53873-53881.

51. Shcherbinin, D.P.; Konshina, E.A. Impact of titanium dioxide nanoparticles on purification and contamination of nematic liquid crystals. Beilstein J. Nanotechnol. 2017, 8, 2766-2770.

52. Kovalchuk, O.V.; Studenyak, I.P.; Izai, V.Y.; Rubak, S.O.; Pogodin, A.I.; Kopcansky, P.; Timko, M.; Gdovinova, V.; Mariano, J.; Kovalchuk, T.M. Saturation effect for dependence of the electrical conductivity of planar oriented liquid crystal 6CB on the concentration of Cu7PS6 nanoparticles. Semicond. Phys. Quantum Electron. Optoelectron. 2017, 20, 437-441.

53. Shcherbinin, D.P.; Konshina, E.A. Ionic impurities in nematic liquid crystal doped with quantum dots CdSe/ZnS. Liq. Cryst. 2017, 44, 648-655.

54. Konshina, E.; Shcherbinin, D. Comparison of the properties of nematic liquid crystals doped with $\mathrm{TiO}_{2}$ and CdSe/ZnS nanoparticles. J. Mol. Liq. 2017, doi:10.1016/j.molliq.2017.12.112.

55. Shukla, R.K.; Liebig, C.M.; Evans, D.R.; Haase, W. Electro-optical behaviour and dielectric dynamics of harvested ferroelectric $\mathrm{LiNbO}_{3}$ nanoparticle-doped ferroelectric liquid crystal nanocolloids. RSC Adv. 2014, 4, 18529-18536.

56. Basu, R.; Garvey, A. Effects of ferroelectric nanoparticles on ion transport in a liquid crystal. Appl. Phys. Lett. 2014, 105, 151905.

57. Garbovskiy, Y.; Glushchenko, I. Ion trapping by means of ferroelectric nanoparticles, and the quantification of this process in liquid crystals. Appl. Phys. Lett. 2015, 107, 041106.

58. Hsiao, Y.G.; Huang, S.M.; Yeh, E.R.; Lee, W. Temperature-dependent electrical and dielectric properties of nematic liquid crystals doped with ferroelectric particles. Displays 2016, 44, 61-65.

59. Al-Zangana, S.; Turner, M.; Dierking, I. A comparison between size dependent paraelectric and ferroelectric $\mathrm{BaTiO}_{3}$ nanoparticle doped nematic and ferroelectric liquid crystals. J. Appl. Phys. 2017, 121, 085105.

60. Kumar, P.; Debnath, S.; Rao, N.V.S.; Sinha, A. Nanodoping: A route for enhancing electro-optic performance of bent core nematic system. J. Phys. Condens. Matter 2018, 30, 095101.

61. Garbovskiy, Y. Switching between purification and contamination regimes governed by the ionic purity of nanoparticles dispersed in liquid crystals. Appl. Phys. Lett. 2016, 108, 121104.

62. Garbovskiy, Y. Electrical properties of liquid crystal nano-colloids analysed from perspectives of the ionic purity of nano-dopants. Liq. Cryst. 2016, 43, 648-653.

63. Garbovskiy, Y. Impact of contaminated nanoparticles on the non-monotonous change in the concentration of mobile ions in liquid crystals. Liq. Cryst. 2016, 43, 664-670.

64. Garbovskiy, Y. Ions and size effects in nanoparticle/liquid crystal colloids sandwiched between two substrates. The case of two types of fully ionized species. Chem. Phys. Lett. 2017, 679, 77-85.

65. Garbovskiy, Y. Nanoparticle enabled thermal control of ions in liquid crystals. Liq. Cryst. 2017, 44, 948-955.

66. Garbovskiy, Y. Ions in liquid crystals doped with nanoparticles: Conventional and counterintuitive temperature effects. Liq. Cryst. 2017, 44, 1402-1408. 
67. Garbovskiy, Y. The purification and contamination of liquid crystals by means of nanoparticles. The case of weakly ionized species. Chem. Phys. Lett. 2016, 658, 331-335.

68. Garbovskiy, Y. Ion capturing/ion releasing films and nanoparticles in liquid crystal devices. Appl. Phys. Lett. 2017, 110, 041103.

69. Garbovskiy, Y. Kinetics of Ion-Capturing/Ion-Releasing Processes in Liquid Crystal Devices Utilizing Contaminated Nanoparticles and Alignment Films. Nanomaterials 2018, 8, 59, doi:10.3390/nano8020059.

70. Garbovskiy, Y. Adsorption of ions onto nanosolids dispersed in liquid crystals: Towards understanding the ion trapping effect in nanocolloids. Chem. Phys. Lett. 2016, 651, 144-147.

71. Garbovskiy, Y. Nanoparticle-Enabled ion trapping and ion generation in liquid crystals. Adv. Condens. Matter Phys. 2018, 2018, 8914891.

72. Garbovskiy, Y. Adsorption/desorption of ions in liquid crystal nano-colloids: The applicability of the Langmuir isotherm, impact of high electric fields, and effects of the nanoparticle's size. Liq. Cryst. 2016, 43, 853-860.

73. Barbero, G.; Evangelista, L.R. Adsorption Phenomena and Anchoring Energy in Nematic Liquid Crystals; Taylor \& Francis: Boca Raton, FL, USA, 2006.

74. Steffen, V.; Cardozo-Filho, L.; Silva, E.A.; Evangelista, L.R.; Guirardello, R.; Mafra, M.R. Equilibrium modeling of ion adsorption based on Poisson-Boltzmann equation. Colloids Surf. A Physicochem. Eng. Asp. 2015, 468, 159-166.

75. Batalioto, F.; Figueiredo Neto, A.M.; Barbero, G. Ion trapping on silica nanoparticles: Effect on the $\zeta-$ potential. J. Appl. Phys. 2017, 122, 164303.

76. Steffen, V.; Silva, E.A.; Evangelista, L.R.; Cardozo-Filho, L. Debya-Huckel approximation for simplification of ions adsorption equilibrium model based on Poisson-Boltzmann equation. Surf. Interfaces 2018, 10, 144148.

77. Nanowerk Spotlight. Ionic Purity of Nanoparticles Is Key to Switching between Purification and Contamination Regimes in Liquid Crystal Devices. Available online: https://www.nanowerk.com/spotlight/spotid=42995.php (accessed on 30 Mar 2016).

78. Nanowerk Spotlight. A Nanotechnology Approach to Purifying Liquid Crystals. Available online: https://www.nanowerk.com/spotlight/spotid=45659.php (accessed on 23 January 2017).

79. Wu, P.W.; Lee, W. Phase and dielectric behaviors of a polymorphic liquid crystal doped with graphene nanoplatelets. Appl. Phys. Lett. 2013, 102, 162904.

(C) 2018 by the authors. Licensee MDPI, Basel, Switzerland. This article is an open access article distributed under the terms and conditions of the Creative Commons Attribution (CC BY) license (http://creativecommons.org/licenses/by/4.0/). 